\title{
Bump Energy for Durability Prediction of Coil Spring Based on Local Regularity Analysis
}

\author{
C. H. Chin ${ }^{1,2}$, S. Abdullah ${ }^{1 *}$, S. S. K. Singh ${ }^{1}$, A. K. Ariffin ${ }^{1}$, D. Schramm ${ }^{2}$ \\ ${ }^{1}$ Deparrtment of Mechanical and Manufacturing Engineering, Faculty of Engineering \& Built Environment, \\ Universiti Kebangsaan Malaysia, 43600 UKM Bangi, Selangor, MALAYSIA \\ ${ }^{2}$ Departmental Chair of Mechatronics, \\ University of Duisburg-Essen, 47057 Duisburg, GERMANY \\ *Corresponding Author
}

DOI: https://doi.org/10.30880/ijie.2020.12.05.002

Received 8 February 2020; Accepted 14 May 2020; Available online 30 June 2020

\begin{abstract}
This paper aims to study the identification of bumps in vibrational signals and develop bump-energybased durability predictive models for a suspension coil spring. The bump energy of the loading signal is affected by high frequency noises and can lead to inaccurate results. Therefore, it is necessary to eliminate high frequency noise during bump identification. Local regularity analysis was employed to determine the singular points in road signals. Bump signals were then reconstructed from these singular points. Subsequently, bump-energy-based models were developed by correlating with the fatigue lives estimated using the Coffin-Manson, Morrow and Smith-Watson- Topper strainlife models. The results show that the bump signals extracted from the road excitations had a frequency band within 0-50 Hz, indicating that the high frequency noises had been successfully removed during extraction of the bumps. The bump-energy-based models predicted a fatigue life ranging from $3.98 \times 10^{4}$ to $4 \times 10^{9}$ cycles within a $95 \%$ confidence interval, where the Coffin-Manson-based model showed the highest fatigue life. This is because the Coffin-Manson model did not consider the mean stress effects. When compared with the experimental results, the Coffin-Mansonbased model indicates the highest accuracy, given its highest $\mathrm{R}^{2}$ of 0.948 . The bump-energy-based models developed in this study contributed an accurate durability prediction of coil springs.
\end{abstract}

Keywords: Bump extraction, energy, fatigue life, coil spring, regularity analysis

\section{Introduction}

Fatigue failure is often responsible for many component failures in automotive systems. In a suspension system, the coil springs suffer continuous fatigue deterioration owing to the dynamic excitations from the irregular road surfaces $[1,2]$. Fatigue failure of the coil springs can impair the vehicle's control and eventually leads to serious casualties and system failures. Therefore, durability analysis of the coil spring is necessary in the design of the suspension system to ensure the vehicle safety.

In current industrial practices, durability analysis of automotive components relies heavily on time-domain approaches because of their high accuracy. The time-domain approaches compose of the loading histories acquisition, cycle counting of the time series and damage calculation using damage accumulation rule. Among many alternatives, the combination of Rainflow cycle counting and Palmgren-Miner linear damage rule are widely applied because it is simple and accurate [3]. Nonetheless, the application of time-domain approaches on complex real-life systems can be computationally challenging. This is because such approaches require a huge data size to represent the real loading 
conditions [4]. Several efforts had been made by researchers to extract fatigue-related features from the loading signals of mechanical components to accelerate the durability analysis [5,6].

The loading histories of coil spring resulted by road surface excitations often contain some spike-like transients, associated with sudden and large amplitude changes. These transients, also known as 'bumps', are originated from the irregular road surface such as the speed bumps and severe potholes [7]. Researchers supported that the bumps in loading histories are high amplitude cycles that contributed to a major portion of fatigue damage in suspension components especially the coil spring [8,9]. Thus, the extraction of the bump information from the loading signals is very useful for a durability analysis to understand and predict the fatigue behaviour of a component.

Several studies investigated energy content in the loading signals for bumps extraction. For example, several studies $[8,10]$ computed the energy content in loading histories of mechanical components through continuous wavelet transform and regarded the high energy sections as the bumps. Idris et al. [11] also generated energy related parameter for crack propagation prediction. Nonetheless, the bumps in the signals of real-life applications are always immersed in some noises, caused by instrumental or environmental factors. The noises contribute a considerable amount of energy in the signals, regardless their low amplitude ranges. The energy-based bumps identification techniques proposed by previous studies failed to consider the noise effects and this may lead to some serious errors. For this reason, a denoising procedure is necessary prior the bumps extraction to obtain accurate results. Generally, low-pass filter is widely applied to remove the high frequency noises from the loading histories in automotive applications [9]. Nevertheless, the method always requires the users to provide a gate value for the filtering process. The determination of the gate value solely depends on the user's experiences or trial-and-error approach. This renders the filtering process to be less efficient and might cause inacceptable loss of information during the denoising process.

Since the spikes in the road excitations are associated with sudden and large amplitude fluctuations, the spikes can thus be referred as the singularities in the signals. These are the points when a signal is discontinuous and indifferentiable in time. There are scientists who supported that singularities in time series often contain important information that reflects the performance of mechanical systems. Several studies [12,13] successfully applied the regularity analysis on nonstationary time series to identify the singular points for fault detection of mechanical components. Given that the durability of coil spring is closely related to the spikes in the loading histories, local regularity analysis is thus applicable to determine the singular points for denoising and bumps identification.

Noises in the road excitation signals should be removed to identify the bumps and obtain a more accurate fatigue life prediction. This study proposes the local regularity analysis as a denoising and bump identification approach for road excitations of suspension coil spring. In addition, the energy of the extracted bumps from the road excitation signals was used for durability prediction of the coil spring. Numerical models based on the bump energy were developed to simplify the durability assessment of coil springs. The predictive model is expected to provide a simple and accurate durability assessment of the suspension coil springs working under different road conditions. A hypothesis is made in this study: the higher the bump energy in road excitations, the lower the resulted fatigue life.

\section{Methodology}

The methodology included the acquisition of road excitation signals and strain histories of coil spring, bumps extraction from the road excitation signal, determination of bump energy and coil spring fatigue life, development of energy-based fatigue life predictive models and validation of the models. Fig. 1 depicts the methodology flowchart of this study.

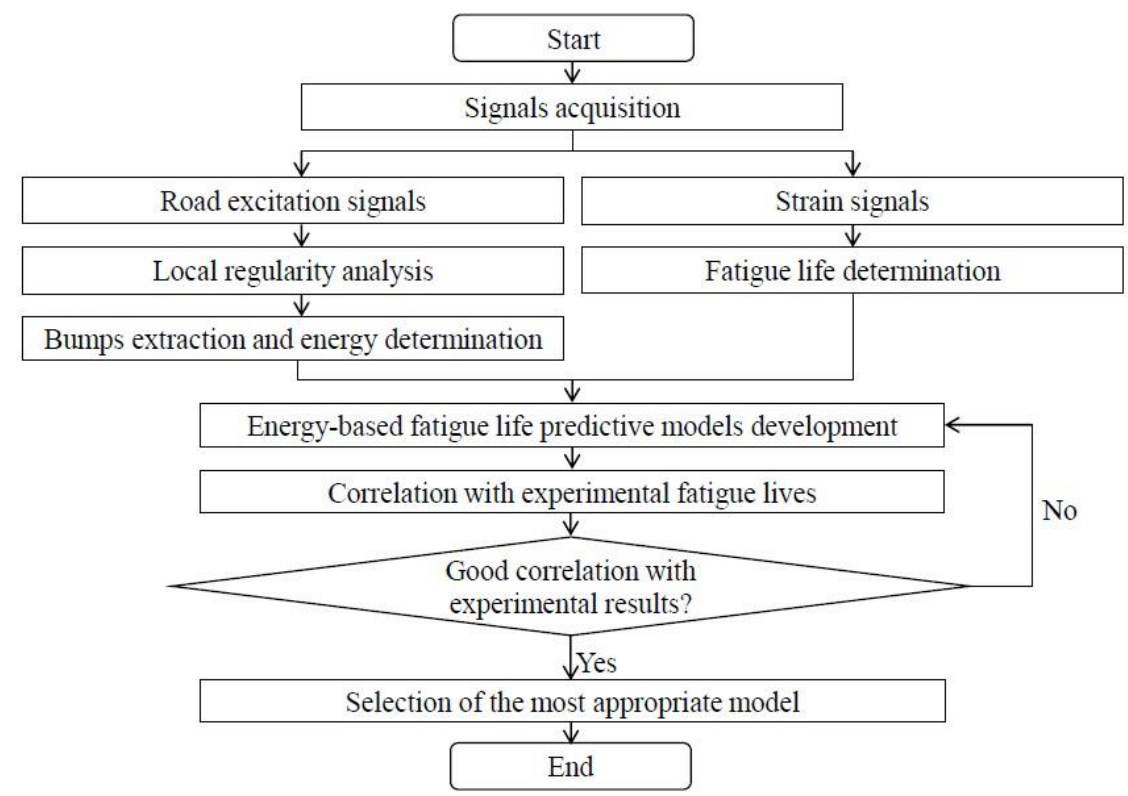

Fig. 1 - Development of bump-energy-based fatigue life predictive model 


\subsection{Road Excitation and Strain Histories Acquisition}

Road tests were performed to acquire road vibrational excitations and strain histories of the suspension system. During the road test, a sedan car with $1300 \mathrm{cc}$ engine capacity was used and its frontal coil spring was tested. The car was equipped with a passive Macpherson suspension system with a spring stiffness of $16,806 \mathrm{~N} / \mathrm{m}$ and a damping coefficient of $15,564 \mathrm{Ns} / \mathrm{m}$. The material of the coil spring is carbon steel SAE5160 which is a common material for coil spring production [14]. The road tests were conducted on several roads with different conditions such as university campus, rural area, highway and newly built road to obtain road excitations with different behaviours.

In current study, the vertical acceleration of car wheel was measured to represent the vibrational loading [15]. To capture the vibrational excitations from the road, an accelerometer was placed on the lower suspension arm. To collect the strain time histories of coil spring, a strain gauge was adhered to the surface of the coil spring. The strain gauge and accelerometer used were shown in Fig. 2. The strain gauge location was determined through the static analysis of the coil spring, the location with highest stress was selected. According to previous study [8], the sampling frequency of data acquisition should be more than $400 \mathrm{~Hz}$ to cover the frequency range of road excitation data. Thus, a sampling frequency of $500 \mathrm{~Hz}$ was employed in current study. The sensors were connected to a data acquisition instruments and a laptop to record the real time acceleration and strain signals simultaneously. Strain and acceleration signals with a total time length of 80 s that consisted of 40,000 data points were collected.

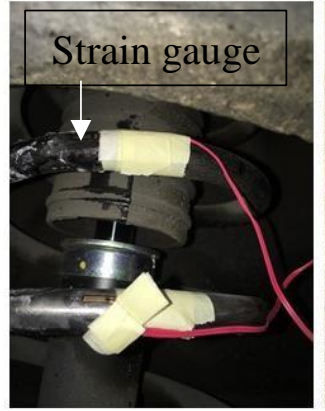

(a)

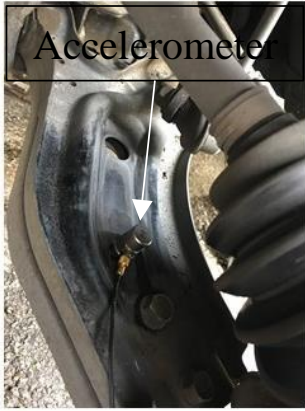

(b)

Fig. 2 - Instruments for signal acquisition: (a) strain gauge and (b) accelerometer

\subsection{Bumps Extraction through Local Regularity Analysis}

In this study, the local regularity of the road excitation time series was described by Hölder continuity. A time series $f(x)$ fulfils the Hölder continuity at $x_{0}$ if there exist a constant $C>0$ and a polynomial $P$ with degree less than $\alpha$ such that:

$$
\left|f(x)-P\left(x-x_{0}\right)\right| \leq C\left|x-x_{0}\right|^{\alpha}
$$

The pointwise Hölder exponent at $x_{0}$ is the supremum of $\alpha$ that fulfils Equation (1). Pointwise Hölder exponent with value less than 1 indicates that the function is nondifferentiable at the point. The nondifferentiable locations in the function are referred as singularities.

To identify the singularities in the time series, wavelet transform maximum modulus (WTMM) method was employed. First, the continuous wavelet transform (CWT) of the road excitation signals was conducted to obtain the timefrequency wavelet coefficients. The CWT is mathematically defined as:

$$
W(s, u)=\left\langle f(t), \psi_{s, u}(t)\right\rangle=\frac{1}{\sqrt{s}} \int_{-\infty}^{+\infty} f(t) \psi^{*}\left(\frac{t-u}{s}\right) d t
$$

where $W(s, u)$ is the wavelet coefficients, $\psi^{*}(s, u)$ is the conjugate of mother wavelet function, $u$ is the location parameter and $s$ is the scale parameter. WTMM are large modulus magnitudes of wavelet coefficients in localised region across the time-scale plane. WTMM reflects the large changes occurred in the signal at specific time point. Thus, the presence of singularities can be determined by the WTMM at specific time point. Relationship between the WTMM and the local Hölder exponent $\alpha$ at time $v$ is given by:

$$
|W(s, u)| \leq A s^{\alpha+1 / 2}
$$

where $|W(s, u)|$ denotes the WTMM and $A$ is a constant. Putting the both sides of Equation (3) in natural logarithm yields: 


$$
\log _{2}|W(s, u)| \leq \log _{2} A+(\alpha+1 / 2) \log _{2} s
$$

Hence, the local Hölder exponent $\alpha$ at time $v$ was numerically estimated from the gradient of the graph $\log _{2}|(s, u)|$ against $\log _{2} S$. Meanwhile, the locations of the singularities in the time series were determined through the time location $v$ where the WTMM converged at finer scale. This study adopted the $2^{\text {nd }}$ derivative of Gaussian $\left(2^{\text {nd }}\right.$ DOG) as the mother wavelet function because the $2^{\text {nd }}$ DOG have two vanishing moments which allows the singularities with Hölder exponents below two to be detected. The DOG wavelet function is given by $[12,13]$ :

$$
\psi(t)=\frac{(-1)^{m+1}}{\sqrt{\Gamma\left(m+\frac{1}{2}\right)}} \frac{d^{m}}{d t^{m}}\left(e^{-t^{2} / 2}\right)
$$

After the locations of singular points were determined, bump signal was obtained by signal reconstruction using linear interpolation method between the singular points. The sampling frequency of the reconstructed signals was maintained at $500 \mathrm{~Hz}$. The reconstructed bump signals were compared with original road excitation signals. Fast Fourier transform (FFT) was also employed to obtain the frequency spectrum of the signals for comparison. The energy content of the bump signals was subsequently computed by summing the square of wavelet coefficients, such that:

$$
E=\sum|W(s, u)|^{2}
$$

\subsection{Establishment of Wavelet-Energy Based Fatigue Life Predictive Model}

The fatigue life of the coil spring was predicted from the strain time histories using strain-life method. This study employed strain-life models because of the localised plastic deformation was significant on fatigue behaviour of the coil spring which had small geometry [15]. The Coffin-Manson, Morrow, and Smith-Watson-Topper (SWT) strain-life models were considered in this study. Coffin-Manson model gives accurate fatigue life prediction with strain histories with zero mean-stress. The Morrow and SWT models are more suitable for fatigue life prediction where mean stress effect is significant. Coffin-Manson, Morrow and SWT strain-life models are given in Equation 7, 8 and 9, respectively:

$$
\begin{aligned}
& \varepsilon_{a}=\frac{\sigma_{f}^{\prime}}{E}\left(2 N_{f}\right)^{b}+\varepsilon_{f}^{\prime} \cdot\left(2 N_{f}\right)^{c} \\
& \varepsilon_{a}=\frac{\left(\sigma_{f}^{\prime}-\sigma_{m}\right)\left(2 N_{f}\right)^{b}}{E}+\varepsilon_{f}^{\prime} \cdot\left(2 N_{f}\right)^{c} \\
& \sigma_{\max } \varepsilon_{a}=\frac{\left(\sigma_{f}^{\prime}\right)^{2}}{E}\left(2 N_{f}\right)^{2 b}+\sigma_{f}^{\prime} \varepsilon_{f}^{\prime} \cdot\left(2 N_{f}\right)^{b+c}
\end{aligned}
$$

where $2 N_{f}$ denotes the reversals of load to failure, $\sigma_{f}^{\prime}$ is the fatigue strength coefficient, $b$ is the fatigue strength exponent ( $b$ has a negative sign), $\varepsilon f$ is the fatigue ductility coefficient, $c$ is the fatigue ductility exponent (the sign of $c$ is negative), $\sigma_{\max }=\sigma_{m}+\sigma_{a}$, is the alternating strain and the mean stress, $m$ is positive for tensile stress and negative for compression stress.

Next, the relationship between the wavelet-energy of bumps in road excitation signals and the durability performance of coil spring was investigated. Ordinary linear regression method was utilised to establish the linear relationship between the variables. It shall be noted that the bumps energy and the fatigue lives of coil spring were transformed into logarithm scale to obtain a linear relationship. This is because the bump energy and fatigue life were found in the range of $10^{9}-10^{11}$ and $10^{4}-10^{10}$, respectively. Thus, putting both variables in logarithm scale could linearize the variables for a better linear regression fitting. The fatigue life predictive linear model was validated by comparing the simulated fatigue life from the linear model to the experimental fatigue life. A correlation study was conducted to confirm the correlation between the predicted and experimental fatigue lives. The $\mathrm{R}^{2}$ values of the models were determined to investigate the relationship between the bump energy and fatigue life. The root-mean-square-errors (RMSE) of the models were also determined to statistically evaluate the accuracy of the models in fatigue life prediction. RMSE was expressed in the following equation:

$$
R M S E=\sqrt{\sum_{i=1}^{n} \frac{\left(N_{\text {factual }}-N_{f \text { simulated }}\right)^{2}}{n}}
$$

where $n$ is the total number of fatigue life. 


\section{Results and Discussion}

\subsection{Establishment of Wavelet-Energy based Fatigue Life Predictive Model}

In this study, the bumps in the road excitation signals were determined using local regularity analysis. Local regularity analysis identified localised singular points in time where the signals were discontinued or indifferentiable. The singular points were then reconstructed using linear interpolation method to obtain signals that consist of only bumps (later known as bump signals). Fig. 3 depicts the original road excitation signals and the corresponding bump signals of different road conditions. Fig. 3 also shows the strain time histories collected at the coil spring. From Fig. 3, it can be seen that the bump signals were identical to the original road signals. The deviation between the bump signals and original signals were evaluated by inspecting the RMSE between the bump and original signals. The RMSE of the road signals from campus, highway, new road and rural were respectively recorded as $0.1464,0.1223,0.0696$ and $0.2133 \mathrm{~g}$. The RMSE values were relatively low compare to the amplitude range of the signals (6-8 g). The low RMSE values indicated that the bumps extracted via local regularity analysis could represent the original signals for durability determination. The rural road showed the highest RMSE because it had the most of road surface irregularities and hence some errors were introduced during the singular points estimation.

The frequency spectrums of the road excitation and bump signals were shown in Fig. 4. The spectrums revealed that the original road excitations had high amplitude peaks in low frequency range between $0-50 \mathrm{~Hz}$. However, the original road excitation signals also composed of some high frequency components above $50 \mathrm{~Hz}$. The campus and rural road showed particularly high peaks at frequency of $80 \mathrm{~Hz}$. After the road excitation signals were processed with the local regularity analysis, it can be observed that only the components in low frequency range of $0-50 \mathrm{~Hz}$ were remained in the bump signals. Previous study [7] remarked that the road excitations are classified as low frequency vibrations, with frequency range between 0 to $50 \mathrm{~Hz}$. This strongly supports that the local-regularity-based bumps identification method proposed in this study is capable to extract bumps signal and eliminate unnecessary high frequency noises. Besides that, it is also noteworthy that the denoising process using local regularity analysis in this study required no input of a gate value from the users. This is because the local regularity analysis was searching for the singular points that represented the discontinuities in the road excitation signals and the bump signals were reconstructed from the singular points. Therefore, the local regularity approach appeared to be a more efficient denoising and bumps identification method compared to the previous methods.
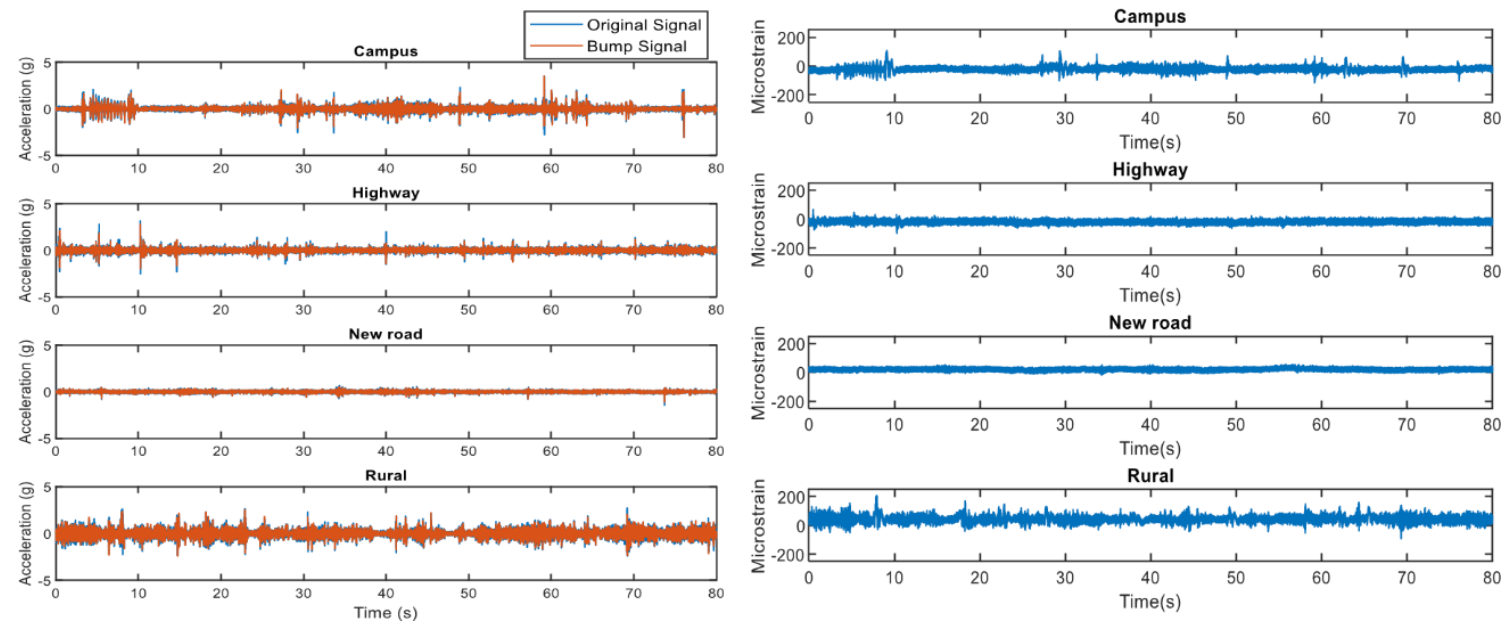

Fig. 3 - Road excitations (left) and strain (right) signals of different road conditions

\subsection{Bump Energy-based Durability Predictive Model}

The total wavelet energy in the bump signals was computed and numerical models for durability prediction were subsequently developed via linear regression method. Fig. 5 shows the fatigue life predictive linear models established based on different strain-life models. The Coffin-Manson, Morrow and SWT linear models are expressed in Equation 11,12 and 13, respectively:

$$
\begin{aligned}
& N_{f}=-4.66(E)+55.06 \\
& N_{f}=-4.123(E)+49.35 \\
& N_{f}=-3.541(E)+43.18
\end{aligned}
$$


where $N_{f}$ denotes the fatigue life and $E$ is the wavelet bump energy. Both $N_{f}$ and $E$ were expressed in logarithm scale of base 10 . The wavelet energy was found to be inversely proportional to the coil spring's fatigue life, as indicated by the negative gradients of the linear models. This is agreed by the previous studies that the high energy cycles contribute to more fatigue damage and result in a shorter fatigue life $[8,10]$. Among the three models, the Coffin-Manson based model had the highest $\mathrm{R}^{2}$ of 0.9506 and the lowest RMSE of 0.5705 . This showed that the Coffin-Manson based model gave the most accurate life prediction of coil spring in 95\% confidence interval. This is because the Coffin-Manson strain-life model offered a good prediction of fatigue life in strain time histories with zero mean value. As shown in Fig. 3, the strain time histories collected at coil spring had mean values close to zero and therefore the Coffin-Manson strain-life model was more suitable to be used.

The simulated fatigue lives from the linear models were correlated to the experimental fatigue lives. Fig. 6 shows the correlation between the simulated fatigue lives and experimental fatigue lives estimated by different strain-life models. Results showed that fatigue lives estimated by Coffin-Manson based linear model had the best correlation to the experimental fatigue lives with the highest $\mathrm{R}^{2}$ value of 0.948 . This indicates that the fatigue lives predicted by the CoffinManson based model had the best 1:1 correlation with the experimental fatigue lives. This additionally proved that the Coffin-Manson based model gave the most accurate fatigue life prediction as indicated by the highest $\mathrm{R}^{2}$ value of the model in Fig. 5.
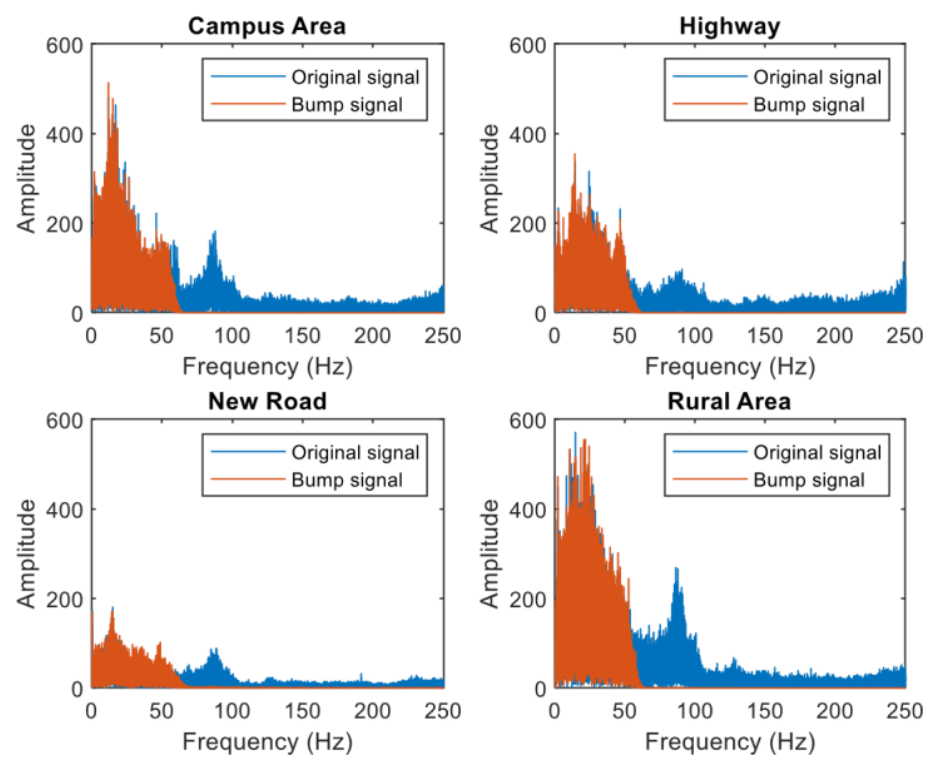

Fig. 4 - Frequency spectrum of original and reconstructed road excitation signals

\section{Conclusion}

In this study, a denoising and bumps extraction method based on local regularity analysis was proposed. This method provided more efficient bumps identification in road excitation signals because this technique required no input of a gate value from the users. The results showed that the local-regularity-based approach can extract bumps in road excitation signals below $50 \mathrm{~Hz}$ while eliminating the high frequency noises. The extracted bump signals closely resembled the original road excitations in the time domain and low frequency band. In addition, the bump energy computed via wavelet analysis was found to have a good fatigue life correlation given their $\mathrm{R}^{2}$ values above 0.8 . The developed bumpenergybased fatigue life predictive model based on the Coffin-Manson strain-life model provides the most accurate durability prediction of the coil spring. The energy-based fatigue life predictive models introduced in this study significantly simplified and accelerated the durability analysis of coil springs subjected to random loading.

\section{Acknowledgement}

Authors are grateful to the support of the Ministry of Education Malaysia and Universiti Kebangsaan Malaysia under the research grant FRGS/1/2015/TK03/UKM/01/2 and the European Unions's Horizon 2020 research and innovation programme under the Marie Sklodowska-Curie grant agreement (No. 730888). 


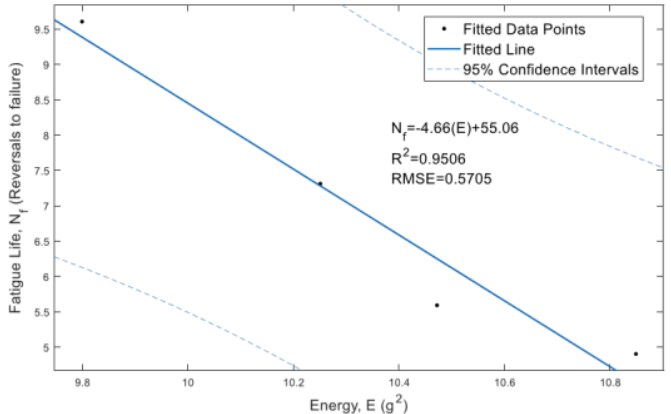

(a)

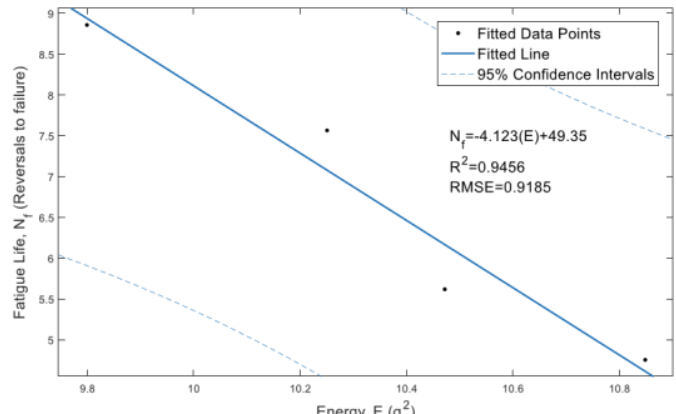

(b)

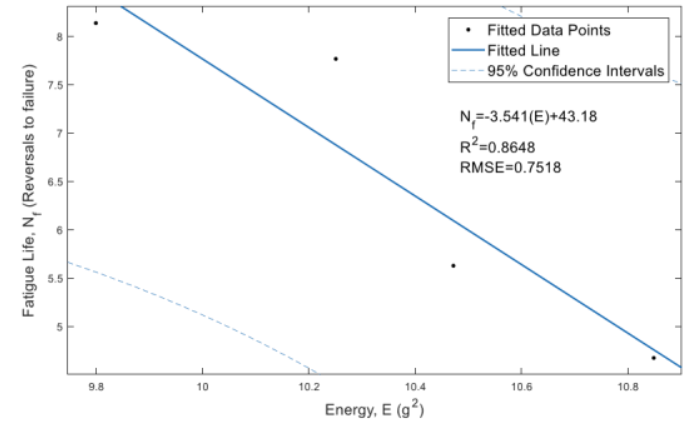

(c)

Fig. 5 - Linear fatigue life predictive model based on different strain-life model: (a) Coffin-Manson; (b) Morrow and (c) Smith-Watson-Topper models

(a)

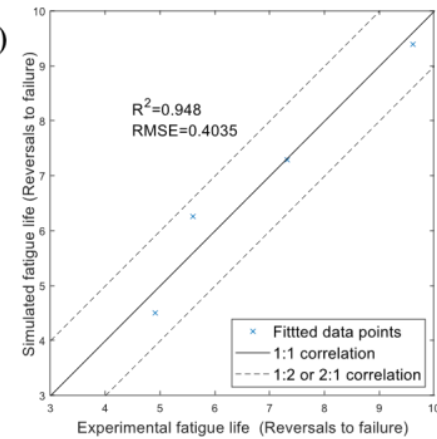

(b)

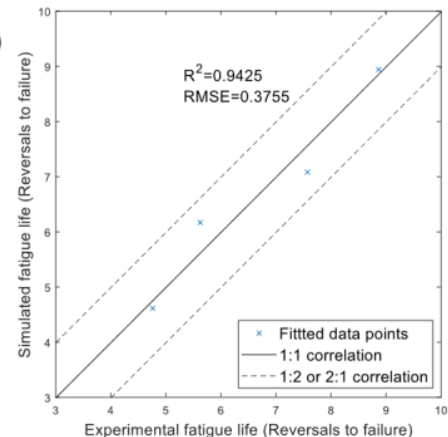

(c)

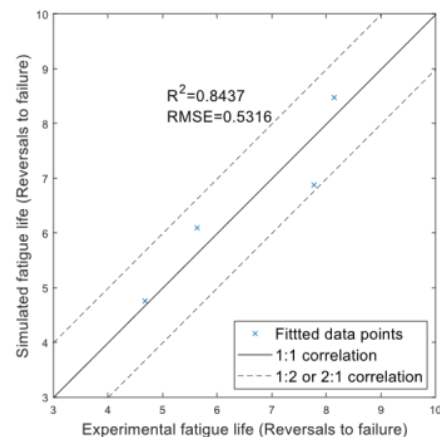

Fig. 6 - Correlations between simulated and experimental fatigue lives based on different strain-life models: (a) Coffin-Manson; (b) Morrow and (c) Smith-Watson-Topper models

\section{References}

[1] Zhu, Y., Wang, Y., \& Huang, Y. (2014). Failure analysis of a helical compression spring for a heavy vehicle's suspension system. Case Studies in Engineering Failure Analysis, 2(2), 169-173.

[2] Vukelic, G., \& Brcic, M. (2016). Failure analysis of a motor vehicle coil spring. Procedia Structural Integrity, 2, 2944-2950.

[3] Altamura, A., \& Straub, D. (2014). Reliability assessment of high cycle fatigue under variable amplitude loading: Review and solutions. Engineering Fracture Mechanics, 121-122, 40-66.

[4] Ugras, R. C., Alkan, O. K., Orhan, S., Kutlu, M., \& Mugan, A. (2019). Real time high cycle fatigue estimation algorithm and load history monitoring for vehicles by the use of frequency domain methods. Mechanical Systems and Signal Processing, 118, 290-304.

[5] Shafiullah, A. K. M., \& Wu, C. Q. (2013). Generation and validation of loading profiles for highly accelerated durability tests of ground vehicle components. Engineering Failure Analysis, 33, 1-16.

[6] Rahim, A. A. A., Singh, S. S. K., Abdullah, S., Nuawi, M. Z. (2018). Strain Signal Characterisation Using the 4th Order of Daubechies Wavelet Transform for Fatigue Life Determination. International Journal of Integrated Engineerig, 10.

[7] Kong, Y. S., Abdullah, S., Schramm, D., Omar, M. Z., Haris, S. M., \& Bruckmann, T. (2017). Mission profiling of road data measurement for coil spring fatigue life. Measurement, 107, 99-100. 
[8] Putra, T. E., Abdullah, S., Schramm, D., Nuawi, M. Z., \& Bruckmann, T. (2017). Reducing cyclic testing time for components of automotive suspension system utilising the wavelet transform and the Fuzzy C-Means. Mechanical Systems and Signal Processing, 90, 1-14.

[9] Duraffourg, S., Argoul, P., Vasseur, E., \& Cumunel, G. (2015). New Spot Weld Fatigue Criteria and Fatigue Data Editing Technique. Procedia Engineering, 133, 433-453.

[10] Pratumnopharat, P., Leung, P. S., \& Court, R. S. (2014). Wavelet transform-based stress-time history editing of horizontal axis wind turbine blades. Renewable Energy, 63, 558-575.

[11] Idris, R., Abdullah, S., Thamburaja, P., Omar, M. Z. (2018). Entropy-Based Approach for Fatigue Crack Growth Rate of Dual-Phase Steel, International Journal of Integrated Engineerig, 10.

[12] Venkatakrishnan, P., Sangeetha, S., Gnanasekaran, J. S., Vishnukumar, M. G., \& Padmanaban, A. S. (2014). Analysis of Vibration in gearbox sensor data using Lipschitz Exponent (LE) function: A Wavelet approach. IFAC Proceedings Volumes, 47(1), 1067-1071.

[13] Zhang, H., Yu, X., Liu, B., Wu, Y., \& Li, Y. (2016). Using wavelets to study spike-type compressor rotating stall inception. Aerospace Science and Technology, 58, 467-479.

[14] Murakami, Y., Kanezaki, T., \& Sofronis, P. (2013). Hydrogen embrittlement of high strength steels: Determination of the threshold stress intensity for small cracks nucleating at nonmetallic inclusions. Engineering Fracture Mechanics, 97, 227-243.

[15] Kong, Y. S., Abdullah, S., Schramm, D., Omar, M. Z., \& Haris, S. M. (2019). Development of multiple linear regression-based models for fatigue life evaluation of automotive coil springs. Mechanical Systems and Signal Processing, 118, 675-695. 